AperTO - Archivio Istituzionale Open Access dell'Università di Torino

On a paper of Berestycki-Hamel-Rossi and its relations to the weak maximum principle at infinity, with applications

This is a pre print version of the following article:

Original Citation:

Availability:

This version is available http://hdl.handle.net/2318/1693253

since 2020-01-08T12:14:35Z

Published version:

DOI:10.4171/RMI/1009

Terms of use:

Open Access

Anyone can freely access the full text of works made available as "Open Access". Works made available under a Creative Commons license can be used according to the terms and conditions of said license. Use of all other works requires consent of the right holder (author or publisher) if not exempted from copyright protection by the applicable law. 


\title{
On a paper of Berestycki-Hamel-Rossi and its relations to the weak maximum principle at infinity, with applications
}

\author{
Marco Magliaro* $\quad$ Luciano Mari ${ }^{\dagger} \quad$ Marco Rigoli ${ }^{\ddagger}$
}

\begin{abstract}
The aim of this paper is to study a new equivalent form of the weak maximum principle for a large class of differential operators on Riemannian manifolds. This new form has been inspired by the work of Berestycki, Hamel and Rossi, [5], for trace operators and allows us to shed new light on it and to introduce a new sufficient bounded Khas'minskii type condition for its validity. We show its effectiveness by applying it to obtain some uniqueness results in a geometric setting.
\end{abstract}

Keywords: weak maximum principle, Khas'minskii type conditions, Lichnerowicz equation, Ricci solitons.

Mathematical subject classification: 58C99, 35B50, 53C21

\section{Introduction}

Maximum principles have numerous interesting applications in analytic and geometric contexts and have therefore been the object of intensive study for many decades. Of particular interest for us are their versions "at infinity", in the spirit of the original works of Omori [16], Yau [26] and Pigola, Setti and the third author [18]. Their geometric applications range, for instance, from the theory of submanifolds in Riemannian and Lorentzian spaces to Ricci and mean curvature solitons. A detailed introduction to the different formulations of the maximum principle and its usefulness in Geometric Analysis can be found in $[2,18]$.

This paper is devoted to the study of a new equivalent form of the weak maximum principle at infinity, WMP for short, first introduced in [17], for a

*Departamento de Matemática, Universidade Federal do Ceará, Fortaleza, Brazil

†Classe di scienze matematiche e naturali, Scuola Normale Superiore, Pisa, Italy

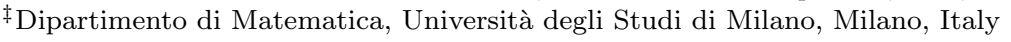


broad family of differential operators on Riemannian manifolds (see Definition 2.1 below). This class includes, for instance, the $p$-Laplacian, the mean curvature operator and others that naturally arise in geometric and analytic settings. The origin of the present work lies in the investigation of a maximum principle formulated in [5], which we now recall. Let $L$ be an elliptic linear differential operator in trace form on $\mathbb{R}^{m}$ written, in the standard basis $\left\{\partial_{i}\right\}$, in the form

$$
L u=a^{i j} u_{i j}+b^{i} u_{i}, \quad a^{i j}, b^{i} \in C_{\mathrm{loc}}^{0, \alpha}\left(\mathbb{R}^{m}\right) .
$$

We recall that the usual strong maximum principle states that for each $c \in$ $L_{\text {loc }}^{\infty}\left(\mathbb{R}^{m}\right)$, any non-positive $C^{2}$ solution of $L u+c(x) u \geq 0$ on an open, connected set $\Omega$ cannot attain the value zero at an interior point unless $u \equiv 0$. This relevant result is due to E. Hopf, and can be found in [23, Thm. 2.1.1] (see also [10, Thm $3.5]$, and [19]). When $\Omega$ has non-compact closure and zero is not attained, one can ask whether or not $u$ may approach zero as $x$ diverges in $\Omega$. The maximum principle in Lemma 2.1 of [5] is stated in the following form: given the open set $\Omega \subseteq \mathbb{R}^{m}$, for each $\varepsilon>0$ and each solution of

$$
\begin{cases}L u+c(x) u \geq \varepsilon & \text { on } \Omega \subset \mathbb{R}^{m} \\ u \leq 0 & \text { on } \Omega\end{cases}
$$

with $\sup _{\partial \Omega} u<0$ if $\partial \Omega \neq \varnothing$ and $c \in C^{0}(\Omega) \cap L^{\infty}(\Omega)$, we have

$$
\sup _{\Omega} u<0
$$

Note that this statement refers to a certain $\Omega$.

Clearly, the validity of this property is not granted for each $L$ and, when $\Omega$ is unbounded, depends on the behavior at infinity of $a^{i j}, b^{i}$ as well as on the growth-decay of the ellipticity constants of $\left\{a^{i j}\right\}$. The search for sharp conditions ensuring (1) is an interesting problem, that has been considered, for instance, in [5], see Lemmas 2.1 and 3.3 therein.

In Theorem 2.2 below, we will prove that a generalized version of the property above on a Riemannian manifold $M$, here called property $(P)$ (see definition 2.3 below) and related to the entire family of open sets $\Omega \subset M$ with $\partial \Omega \neq \varnothing$, is indeed equivalent to the WMP for all the linear and nonlinear operators defined below on $M$. As a consequence, the theory developed in $[2,18]$ for the WMP can be used to deduce very general conditions, e.g. involving only the volume growth of $M$, for the validity of property $(P)$, see for instance Theorem 2.3 below.

The study of the equivalence between the WMP and property $(P)$ presented 
here also enables us to introduce a bounded Khas'minskii-type assumption, sufficient to guarantee the validity of the weak maximum principle. Again, we refer to $[2,14]$ for a thorough presentation of "unbounded" Khas'minskii-type conditions in the linear and nonlinear case, and to [13] for recent improvements.

The terminology is due to the fact that, as proved in [17], the WMP for the Laplace-Beltrami operator is equivalent to the stochastic completeness of the Brownian motion determined by $\Delta$ on $M$, that is, the property that Brownian paths have infinite lifetime almost surely. We also remark that the stochastic completeness of $\Delta$ on $M$ does not require $(M,\langle\rangle$,$) to be geodesically complete$ and, vice versa, the latter does not imply the former.

To show the effectiveness of this new form of the WMP contained in Theorem 2.2 below we prove a comparison, and a companion uniqueness, result for positive bounded solutions of certain differential equations. As a special, remarkable case, we deduce uniqueness for the Lichnerowicz-type equation (see [1] for a detailed introduction)

$$
\Delta u+a(x) u-b(x) u^{\sigma}+c(x) u^{\tau}=0, \quad \tau<1<\sigma
$$

on $M$. More precisely, we have the following

Theorem 1.1. Let $(M,\langle\rangle$,$) be a stochastically complete manifold, a(x), b(x)$, $c(x) \in C^{0}(M) \cap L^{\infty}(M)$ and satisfying $b(x) \geq 0, c(x) \geq 0$ and either

$$
\inf _{M} b(x)>0 \quad \text { or } \quad \inf _{M} c(x)>0 .
$$

Then equation (2) has at most one $C^{2}$ solution $u$ such that for some constant $C>0$,

$$
C^{-1} \leq u \leq C \quad \text { on } M
$$

Remark 1.2. The above theorem is a consequence of the comparison result in Theorem 2.10 below. A similar, but not overlapping, comparison result can be found in Theorem 3.4 of [1], where the assumptions on the coefficients are:

$$
\begin{gathered}
b(x)>0 \text { and } c(x) \geq 0, \\
\sup _{M} \frac{a_{-}(x)}{b(x)}<+\infty, \quad \sup _{M} \frac{c(x)}{b(x)}<+\infty .
\end{gathered}
$$

Remark 1.3. The two-sided bound in (3) is automatically granted under very general conditions on $a, b, c$. See for instance Theorem 3.9 of [1] where the authors give some sufficient conditions.

We note that the condition $c(x) \geq 0$ is quite natural thinking of the physical meaning of the coefficient. Indeed, in the analysis of Einstein's field equations 
in General Relativity, the initial data have to satisfy the Einstein constraint conditions that can be expressed in a geometric form as follows. Let $\left(M^{n}, \widehat{g}\right)$ be a Riemannian manifold of dimension $n \geq 3$ and $\widehat{K}$ a symmetric 2-covariant tensor field on $M$. Then $(M, \widehat{g})$ is said to satisfy the Einstein constraint equations with non-gravitational energy density $\widehat{\rho}$ and non-gravitational momentum density $\widehat{J}$ if

$$
\left\{\begin{array}{l}
S_{\widehat{g}}-|\widehat{K}|_{\widehat{g}}^{2}+\left(\operatorname{tr}_{\widehat{g}} \widehat{K}\right)^{2}=\widehat{\rho} \\
\operatorname{div}_{\widehat{g}} \widehat{K}-\mathrm{d}\left(\operatorname{tr}_{\widehat{g}} \widehat{K}\right)=\widehat{J}
\end{array}\right.
$$

where $S_{\widehat{g}}$ is the scalar curvature of $(M, \widehat{g})$.

A procedure to look for solutions of the above is, according to Lichnerowicz [11], York and Choquet-Bruhat [27, 28, 7], first choosing a conformal data, that is, a Riemannian manifold $\left(M^{n}, g\right)$, a symmetric 2-covariant tensor $\sigma$ for which $\operatorname{tr}_{g} \sigma=0$; a scalar function $\tau$, a nonnegative scalar function $\rho$ and a 1-form $J$. Letting $\Delta, S_{g}$ denote the Laplace-Beltrami operator and the scalar curvature of $g$, one then looks for a function $u>0$ and a vector field $W$ that solve, in the case of an Einstein-scalar field, the system

$$
\left\{\begin{array}{l}
\Delta u-c_{m} S_{g} u+c_{m}\left[\left|\sigma+\stackrel{\circ}{\mathcal{L}}_{W} g\right|_{g}^{2}+\rho\right] u^{-2^{*}-1}-b_{m} \tau^{2} u^{2^{*}-1}=0 \\
\operatorname{div}_{g}\left(\sigma+\dot{\circ}_{W} g\right)=J+\frac{n-1}{n} u^{2^{*}} \mathrm{~d} \tau
\end{array}\right.
$$

where $\stackrel{\circ}{\mathcal{L}}_{W} g$ is the traceless Lie derivative of $g$ in the direction $W$, and

$$
2^{*}=\frac{2 m}{m-2}, \quad c_{m}=\frac{m-2}{4(m-1)}, \quad b_{m}=\frac{m-2}{4 m} .
$$

If $(u, W)$ is a solution of $(5)$, then setting

$$
\widehat{g}=u^{\frac{4}{n-2}} g, \quad \widehat{K}=u^{-2}\left(\sigma+\stackrel{\circ}{\mathcal{L}}_{W} g\right)+\frac{\tau}{n} u^{\frac{4}{n-2}} g, \quad \widehat{\rho}=\rho u^{-2 \cdot 2^{*}}, \quad \widehat{J}=J u^{-2^{*}}
$$

the 4-tuple $(\widehat{g}, \widehat{K}, \widehat{\rho}, \widehat{J})$ solve (4). For details we refer to [6]. Evidently, the scalar equation in (5) is of the form (2) with $c(x) \geq 0, b(x) \geq 0$.

Further comments on Theorem 1.1, for instance on assumption (3), will be given in Section 3.

As a second example, let us consider the operator

$$
\Delta_{f}=\Delta-\langle\nabla f, \nabla\rangle, \quad f \in C^{\infty}(M) .
$$

This operator naturally appears in the study of gradient Ricci solitons, that is, Riemannian manifolds $(M,\langle\rangle$,$) with a potential function f$, if any, satisfying 
the equation

$$
\operatorname{Ricc}+\operatorname{Hess}(f)=\lambda\langle,\rangle
$$

for some constant $\lambda \in \mathbb{R}$. Solitons generate self-similar solutions of the Ricci flow and they are said to be expanding, steady or shrinking respectively when $\lambda<0, \lambda=0, \lambda>0$. We have the following

Theorem 1.4. Let $(M,\langle\rangle$,$) be a complete manifold with a fixed origin o and$ set $r(x)=\operatorname{dist}(x, o)$. Let $f \in C^{\infty}(M)$ and suppose that

$$
\liminf _{r \rightarrow+\infty} \frac{\log \int_{B_{r}} e^{-f}}{r^{2}}<+\infty .
$$

Let $a(x), b(x) \in C^{0}(M) \cap L^{\infty}(M)$ satisfy

$$
\inf _{M} b(x)>0
$$

Then the equation

$$
\Delta_{f} u+a(x) u-b(x) u \log u=0
$$

admits at most one solution $u \in C^{2}(M)$ satisfying $C^{-1} \leq u \leq C$ on $M$, for some $C>0$.

Proof (of Theorem 1.4). We note that under assumption (7) we have the validity of the WMP for the operator $\Delta_{f}$, see [2], chapter 4. The remaining assumptions guarantee the applicability of Theorem 2.10, from which the result follows at once.

Corollary 1.5. Let $(M,\langle\rangle$,$) be a stochastically complete manifold, a, b \in \mathbb{R}$ with $b>0$. Then the only solution $u$ of

$$
\Delta u+a u-b u \log u=0
$$

satisfying $C^{-1} \leq u \leq C$ on $M$, for some $C>0$, is $u=e^{\frac{a}{b}}$.

Suppose now that $(M,\langle\rangle, f$,$) is a gradient soliton. By Hamilton's identity,$

$$
S+|\nabla f|^{2}-2 \lambda f=\Lambda
$$

for some constant $\Lambda \in \mathbb{R}$ and where $S$ is the scalar curvature of $M$. Tracing (6)

$$
S+\Delta f=m \lambda, \quad m=\operatorname{dim} M,
$$

so that putting the two equations together we obtain

$$
\Delta f-|\nabla f|^{2}+2 \lambda f=m \lambda-\Lambda \text {. }
$$


We set $u=e^{-f}$ and the above becomes

$$
\Delta u+(m \lambda-\Lambda) u+2 \lambda u \log u=0 .
$$

Corollary 1.6. Let $(M,\langle\rangle$,$) be a stochastically complete manifold with non$ constant scalar curvature. Then there are no expanding Ricci soliton structures on $M$ with bounded potential.

Remark 1.7. If $(M,\langle\rangle$,$) is geodesically complete, by Proposition 8.7$ and 8.12 of [2] a gradient Ricci soliton structure on $(M,\langle\rangle$,$) automatically implies that$ $M$ is stochastically complete. As a matter of fact, the full Omori-Yau principle holds both for $\Delta$ and for $\Delta_{f}=\Delta-\langle\nabla f, \nabla\rangle$, as shown in [9].

The paper is organized as follows: Section 2 is devoted to the introduction of the family of differential operators, the weak maximum principle and the statement of the aforementioned conditions for its validity, while Section 3 contains the proofs of the results stated in the previous section.

\section{The weak maximum principle}

In what follows we denote by $(M,\langle\rangle$,$) a connected Riemannian manifold of$ dimension $m \geq 2$. Let $X$ be a smooth vector field on $M$ and $T$ be a symmetric, 2-covariant, positive semidefinite tensor field. With $t: T M \rightarrow T M$ we indicate the corresponding endomorphism defined by

$$
t(Y)=T(Y, \quad)^{\sharp}
$$

where ${ }^{\sharp}: T^{*} M \rightarrow T M$ is the musical isomorphism.

We let $\varphi: M \times \mathbb{R}_{0}^{+} \rightarrow \mathbb{R}_{0}^{+}$satisfy

$$
\begin{array}{llll}
\varphi(, s) \in C^{0}(M) & \forall s \in \mathbb{R}_{0}^{+} ; & \varphi(x,) \in C^{0}\left(\mathbb{R}_{0}^{+}\right) & \forall x \in M ; \\
\varphi(x, 0)=0 & \forall x \in M ; & \varphi(x, s)>0 & \text { on } M \times \mathbb{R}^{+} .
\end{array}
$$

More requirements will be added in due time. We define the operator $Q=$ $Q_{\varphi, X, T}$ acting on $C^{1}(M)$ by

$$
Q u=\operatorname{div}\left(|\nabla u|^{-1} \varphi(x,|\nabla u|) t(\nabla u)\right)-\langle X, \nabla u\rangle
$$

in the appropriate weak sense. Of course the definition can be extended to a larger class of functions but in what follows we restrict ourselves to $C^{1}(M)$. Note that for $\varphi(x, s)=s$ and $u \in C^{2}(M)$ the previous operator can also be put 
into the form

$$
Q u=\operatorname{tr}(t \circ \operatorname{hess}(u))+\left\langle(\operatorname{div} T)^{\sharp}-X, \nabla u\right\rangle,
$$

where hess $(u)$ is the $(1,1)$-version of the Hessian of $u$. Thus, with the appropriate choice of $X$, linear trace operators are included in the family (9) (at least when $T$ is $C^{1}$ ). Other interesting examples are obtained with the choices

$$
\begin{cases}(\varphi, t, X)=\left(s^{p-1}, I, 0\right), p>1 & \text { the } p \text {-Laplace operator } \\ (\varphi, t, X)=\left(\frac{s}{\sqrt{1+s^{2}}}, I, 0\right) & \text { the mean curvature operator } \\ (\varphi, t, X)=(s, I, X) & \text { the } X \text {-Laplacian. }\end{cases}
$$

The last example is ubiquitous in the theory of Ricci solitons, especially in the gradient case $X=\nabla f$ for some potential function $f \in C^{\infty}(M)$. Moreover, the same kind of operator is also key in the study of optimal transportation ([25]) and solitons for the mean curvature flow ([8]).

Another example deserves special attention. Let $f: M \rightarrow N$ be a two-sided immersed hypersurface with a chosen, global unit normal vector field $\nu$. Set $A$ to denote the second fundamental tensor in the direction of $\nu$. For $0 \leq k \leq m$ Newton's operators $P_{k}: T M \rightarrow T M$ are inductively defined by setting

$$
P_{0}=I, \quad P_{k}=S_{k} I-A \circ P_{k-1},
$$

where $S_{k}$ is the $k$-th symmetric function of the eigenvalues of $A$ with the agreement that $S_{0}=1$. The associated differential operators acting on $C^{2}(M)$

$$
L_{k} u=\operatorname{tr}\left(P_{k} \circ \operatorname{hess}(u)\right)
$$

are of the form (9) with the choice $X=\operatorname{div} P_{k}$. Note that the ellipticity of $L_{k}$ is not automatically granted, but there exist various sufficient conditions of a geometric nature; see for instance the discussion in Section 3.2 of [3] and the references therein.

We begin by recalling the definition of the weak maximum principle.

Definition 2.1. We say that the weak maximum principle holds for $Q$ on $M$ if for each $u \in C^{1}(M)$ with $u^{*}=\sup _{M} u<+\infty$ and for each $\gamma<u^{*}$ we have

$$
\inf _{\Omega_{\gamma}} Q u \leq 0,
$$

where

$$
\Omega_{\gamma}=\{x \in M: u(x)>\gamma\}
$$


Of course, (10) has to be interpreted in the appropriate weak sense, that is, for each $\varepsilon>0$ there exists $\psi \in C_{c}^{\infty}\left(\Omega_{\gamma}\right), \psi \geq 0, \psi \neq \equiv 0$, such that

$$
-\int|\nabla u|^{-1} \varphi(x,|\nabla u|) T(\nabla u, \nabla \psi)+\langle X, \nabla u\rangle \psi \leq \varepsilon \int \psi .
$$

Before defining property $(P)$ for the operator $Q$ in possibly unbounded domains, it is worth to comment on the quasilinear version of E. Hopf's strong maximum principle recalled in the Introduction. To the best of our knowledge, a strong maximum principle for inequalities of the type

$$
Q u \geq B(x, u, \nabla u) \quad \text { on } \Omega \subset M,
$$

that is, the statement that each non-constant, $C^{1}$-solutions $u \leq 0$ of (11) cannot attain the value zero, first appeared in a generality close to the one of the present paper in [22, Thm. 8.1] (see also [21]). The conditions placed on $Q$ and $B$ are mild, in particular, they do not require $B$ to be increasing in the variable $u$. Hence, they correspond in the linear case $L u+c(x) u \geq 0$ to coefficients $c(x)$ which might be positive somewhere. For related results in a manifold setting, see [20, Thms. 5.5, 5.6]. Section 5.4 in [23] contains a thorough discussion of the strong maximum principle for (11), together with various interesting comments.

We now proceed to give the definition of property $(\mathrm{P})$, mentioned in the introduction, and to make explicit its relation to the WMP:

Definition 2.2. A pair of functions $(c(x), h(s)) \in L_{\mathrm{loc}}^{\infty}(M) \times C^{0}(\mathbb{R})$ is called admissible for property $(P)$ below if $h(0)=0$.

Definition 2.3. We say that $Q$ satisfies property $(P)$ for the admissible pair $(c, h)$ if, for each open set $\Omega \subset M$ with $\partial \Omega \neq \varnothing$, for each $0<\beta(s) \in C^{0}(\mathbb{R})$, each solution $v \in C^{0}(\bar{\Omega}) \cap C^{1}(\Omega)$ of

$$
\begin{cases}Q v+c(x) h(v) \geq \beta(v) & \text { on } \Omega \\ v \leq 0 & \text { on } \Omega \\ \sup _{\partial \Omega} v<0, & \end{cases}
$$

satisfies $\sup v<0$.

Remark 2.1. Clearly, the maximum principle in [5], with a fixed $\Omega \subseteq \mathbb{R}^{m}$, can be recovered if $T$ has components $\left\{a^{i j}\right\}, X=X^{i} \partial_{i}$ with $X^{i}=T_{k k}^{i}-b^{i}$, $\varphi(x, s)=s, h(s)=s$ and $\beta(s)=\varepsilon>0$. However, we note that $h(s)$ does not need to have the same sign of $s$.

We are now in a position to state our first main result: 
Theorem 2.2. Let $(M,\langle\rangle$,$) be a Riemannian manifold, let \varphi: M \times \mathbb{R}_{0}^{+} \rightarrow \mathbb{R}_{0}^{+}$ satisfy ( $\varphi 1)$ and let $X, T$ be as above. Consider the operator $Q$ defined in (8). Then the following statements are equivalent:

i) $Q$ satisfies the WMP on $M$.

ii) $Q$ satisfies (P) for each admissible pair $(c, h)$ with $c_{-} \in L^{\infty}(M)$ and $\operatorname{sh}(s) \geq 0$ on $\mathbb{R}$.

iii) $Q$ satisfies $(P)$ for each admissible pair $(c, h)$ with $c \in L^{\infty}(M)$.

iv) $Q$ satisfies $(P)$ for some admissible pair $(c, h)$ with $c \in L^{\infty}(M)$.

Here, as usual, $c_{-}(x)=-\min \{0, c(x)\}$ is the negative part of $c$.

Despite the simplicity of the proof, the above equivalences are particularly interesting since there are various sufficient conditions for $Q$ to satisfy the WMP. Among them, a very general criterion is the following one, that can be deduced from Theorem 4.1 of [2]: suppose that $X \equiv 0$ and

$$
\varphi(x, s) \leq A(x) s^{\delta} \quad \text { on } M \times \mathbb{R}_{0}^{+}
$$

for some $\delta>0$ and $A(x) \in C^{0}(M)$ with $A(x)>0$ on $M$. Fix an origin $o \in M$ and set $r(x)=\operatorname{dist}(x, o)$. Let $B_{r}$ denote the geodesic ball of radius $r$ centered at $o$. Let $T$ be a 2-covariant, symmetric tensor field on $M$ satisfying

$$
\text { there exist } T_{ \pm} \in C^{0}\left(\mathbb{R}_{0}^{+}\right) \text {such that } 0<T_{-}(r) \leq T(Y, Y) \leq T_{+}(r)
$$

for each $Y \in T_{x} M,|Y|=1, x \in \partial B_{r}$. According to the value of $\delta$ introduced in $(\varphi 2)$, define

$$
T_{\delta}(r)= \begin{cases}T_{+}(r) & \text { if } 0<\delta \leq 1 \\ T_{-}(r)^{\frac{1-\delta}{2}} T_{+}(r)^{\frac{1+\delta}{2}} & \text { if } \delta>1\end{cases}
$$

and

$$
T_{\delta}^{*}(r)=\max _{[0, r]} T_{\delta}(s) .
$$

Theorem 2.3. Let $(M,\langle\rangle$,$) be a complete Riemannian manifold with a fixed$ origin o, and let $\varphi: M \times \mathbb{R}_{0}^{+} \rightarrow \mathbb{R}_{0}^{+}$satisfy $(\varphi 1),(\varphi 2)$. With $A(x)$ and $\delta$ as in $(\varphi 2)$ assume that

$$
A(x) \leq \mathscr{A}(r(x))
$$

where $\mathscr{A}: \mathbb{R}_{0}^{+} \rightarrow \mathbb{R}^{+}$is continuous, non decreasing. Let $T$ satisfy (13) and suppose

$$
\lim _{r \rightarrow+\infty} \frac{T_{\delta}^{*}(r) \mathscr{A}(r)}{r^{1+\delta}}=0
$$




$$
\liminf _{r \rightarrow+\infty} \frac{T_{\delta}^{*}(r) \mathscr{A}(r)}{r^{1+\delta}} \log \left(\int_{B_{r}} A(x)\right)<+\infty .
$$

Then the operator $Q$ defined in (8) with $X \equiv 0$ satisfies each of $i), \ldots, i v)$ in Theorem 2.2.

Example 2.4. If $Q$ is a linear operator with $X \equiv 0$ (for which $\varphi(x, s)=s$ and thus $\mathscr{A}(r) \equiv 1)$, and the biggest eigenvalue of $T$ satisfies $T_{+}(r) \leq C(1+r)^{\mu}$ for some $C>0, \mu \geq 0$, then property $(P)$ is met whenever $\mu<2$ and

$$
\liminf _{r \rightarrow+\infty} \frac{\log \operatorname{Vol}\left(B_{r}\right)}{r^{2-\mu}}<+\infty
$$

This last condition is certainly satisfied, for instance, on $\mathbb{R}^{m}$ for each $m \geq 1$. Consequently, a self-adjoint, linear operator on $\mathbb{R}^{m}$ as in (9) with $X \equiv 0$ satisfies the WMP (hence, the principle in [5] for every fixed $\Omega \subseteq \mathbb{R}^{m}$ ) whenever $T_{+}(r) \leq$ $C(1+r)^{\mu}$ and $\mu<2$.

We would like to note that there are many natural geometric contexts where $(\varphi 2)$ is satisfied. For instance if $\left(M,\langle\rangle,, e^{-f} d V_{M}\right), f \in C^{\infty}(M)$, is a weighted Riemannian manifold and $\varphi: \Sigma \rightarrow M$ is an isometric immersion, one defines the weighted mean curvature vector $H_{f}$ by setting $H_{f}=H+(\bar{\nabla} f)^{\perp}$, where $\bar{\nabla}$ is the gradient on $M$ and $H$ is the usual, non-normalized, mean curvature vector. Given a relatively compact domain $\Omega \subset \Sigma$, the weighted volume of $\Omega$ is given by

$$
\operatorname{Vol}_{f}(\Omega)=\int_{\Omega} e^{-f} d V_{\Sigma}
$$

Then $\Sigma$ is $f$-minimal, that is, a critical point of the weighted volume functional with respect to compactly supported variations if and only if $H_{f} \equiv 0$.

The Jacobi operator $L_{f}$ of an $f$-minimal hypersurface is given by

$$
e^{-f} L_{f} u=\operatorname{div}\left(e^{-f} \nabla u\right)+e^{-f}\left(|A|^{2}+\overline{\operatorname{Ricc}}_{f}(\nu, \nu)\right) u,
$$

where $A$ is the second fundamental tensor of the immersion in the direction of the unit normal vector $\nu$ determining the orientation of $\Sigma$ and $\overline{\operatorname{Ricc}}_{f}=$ $\overline{\operatorname{Ricc}}+\operatorname{Hess}(f)$, is the Bakry-Emery Ricci tensor of $M$. In this case

$$
\varphi(x, s)=e^{-f(x)} s,
$$

that clearly satisfies $(\varphi 2)$ with $\delta=1, A(x)=e^{-f(x)}>0$.

We observe that the above example includes that of (for instance) selfshrinkers in $\mathbb{R}^{n}$, that is, immersions $\varphi: M^{m} \rightarrow \mathbb{R}^{n}$ for which the (not nor- 
malized) mean curvature vector satisfies

$$
H=-\frac{1}{2} \varphi^{\perp}
$$

To see this it is enough to choose $f(x)=\frac{|x|^{2}}{4}$ on $\mathbb{R}^{n}$. We refer the interested reader to [12] for an introduction to the mean curvature flow and the role of selfshrinkers as models for its singularities, and to [8] for deep results that exploit the parallelism between self-shrinkers and $f$-minimal hypersurfaces.

Vice versa, a sufficient condition for $Q$ to satisfy $(P)$ will provide a sufficient condition for $Q$ to satisfy the WMP on $M$. In this way we are able to introduce a "bounded" Khas'minskii-type condition in Definition 2.4 below. We observe that a sufficient Khas'minskii-type assumption via a function $\gamma$ (in the linear case) or a family of functions $\left\{\gamma_{\varepsilon}\right\}_{\varepsilon>0}$ (in the nonlinear case) on $M$ have already been given for linear and nonlinear operators, and we refer to [2] and [14] for a thorough discussion. However, the validity of the latter results rests on the property that

$$
\gamma(x), \gamma_{\varepsilon}(x) \rightarrow+\infty \quad \text { as } x \rightarrow \infty \text { in } M
$$

In the assumptions we are going to present here the function $\gamma$ is bounded. Indeed, we introduce the following condition that we name the "bounded Khas'minskii" property, $(B K)$ for short.

Definition 2.4. We say that $Q$ satisfies property $(B K)$ for some admissible pair $(c, h)$ if, for each $p \in M$ and each $\varepsilon>0$ there exist constants $B_{1,2}=B_{1,2}(\varepsilon)>0$ and a function $\gamma=\gamma_{p, \varepsilon} \in C^{1}(M)$ with the following properties:

$$
\begin{cases}i) & \gamma(p)=0 \\ i i) & 0 \leq \gamma \leq B_{1} \text { on } M \\ i i i) & Q \gamma+c(x) h(\gamma) \leq \varepsilon \\ i v) & \liminf _{x \rightarrow \infty} \gamma(x) \geq B_{2}\end{cases}
$$

We suggest that the reader consult the recent [13] for a detailed study of the interplay between $(B K)$ and previous Khas'minskii conditions. The aforementioned paper deals with classes of operators that include most (though not all) of those considered in the present work, and the main problems are tackled via the use of viscosity solutions.

In the next results we shall need a few more technical requirements to guarantee the ellipticity of $Q$ :

$$
\varphi(x, \quad) \in C^{0}\left(\mathbb{R}_{0}^{+}\right) \cap C^{1}\left(\mathbb{R}^{+}\right) \quad \text { for each } x \in M
$$




$$
\begin{gathered}
\frac{\partial \varphi}{\partial s}(x, \quad)>0 \text { on } \mathbb{R}^{+} \text {for each } x \in M ; \\
\frac{\varphi(x, s)}{s} \in L^{1}\left(0^{+}\right) \text {for each } x \in M ;
\end{gathered}
$$

for each $x \in M, 0 \neq \xi \in T_{x} M$, the (symmetric) bilinear form

$$
\frac{1}{|\xi|^{2}}\left\{\frac{\partial \varphi}{\partial s}(x,|\xi|)-\frac{\varphi(x,|\xi|)}{|\xi|}\right\}\langle\xi,\rangle \odot T(\xi, \quad)+\frac{\varphi(x,|\xi|)}{|\xi|} T(, \quad)
$$

is positive definite.

Here $\odot$ denotes the symmetric tensor product $a \odot b=\frac{1}{2}(a \otimes b+b \otimes a)$.

Remark 2.5. In the linear case $\varphi(x, s)=s,(T 1)$ implies that $T$ be positive definite.

We are now ready to state our second main result, which relates condition $(B K)$ to the WMP and property $(P)$.

Theorem 2.6. Let $(M,\langle\rangle$,$) be a Riemannian manifold and \varphi, X, T$ be as above. Assume the validity of $(\varphi 1),(\varphi 3),(\varphi 4),(\varphi 5),(T 1)$ and of $(B K)$ for an admissible pair $(c, h)$ with

$$
c_{-} \in L^{\infty}(M), \quad \operatorname{sh}(s) \geq 0 \text { on } \mathbb{R} .
$$

Then $(P)$ holds for that pair. In particular, if also $c \in L^{\infty}(M)$ or $(B K)$ holds for the pair $(0,0)$, then $Q$ satisfies the WMP and $(P)$ holds for each admissible pair $(c, h)$ with $c \in L^{\infty}(M)$.

Conditions $(\varphi 3),(\varphi 4),(\varphi 5),(T 1)$ are needed to ensure the validity of a comparison theorem for the divergence part $Q_{0}$ of $Q$, that is, for the operator

$$
Q_{0} u=\operatorname{div}\left(|\nabla u|^{-1} \varphi(x,|\nabla u|) t(\nabla u)\right)
$$

Since the result is interesting in its own, we report its statement here referring to Section 3 for its proof. We stress that the extra assumptions $(\varphi 3),(\varphi 4)$, $(\varphi 5),(T 1)$ are mild, making the comparison very general and, to the best of our knowledge, new.

Proposition 2.7. Assume that $(\varphi 1),(\varphi 3),(\varphi 4),(\varphi 5),(T 1)$ hold and let $\Omega \subset$ $M$ be a relatively compact domain. Let $u, v \in C^{0}(\bar{\Omega}) \cap C^{1}(\Omega)$ solve

$$
\begin{cases}Q_{0} u \geq Q_{0} v & \text { on } \Omega \\ u \leq v & \text { on } \partial \Omega\end{cases}
$$


where $Q_{0}$ is the operator defined in (14). Then, $u \leq v$ on $\Omega$.

Remark 2.8. It shall be noticed that Theorems 5.2 and 5.3 in [20] give sharp comparison results for inequalities of the type

$$
Q_{0} u \geq B(x, u, \nabla u), \quad Q_{0} v \leq B(x, v, \nabla v),
$$

where $B, u, v$ satisfy suitable assumptions. However, the results are skew with Proposition 2.7. The monograph [23] contains a thorough investigation of comparison principles for a variety of differential operators, including operators in divergence form (although under more restrictive assumptions), and is by now a standard reference for the interested reader. See also $[22,4]$ for improvements and other related results.

When the comparison property for $Q_{0}$ in the form of Proposition 2.7 above is available, in order to prove the validity of the WMP it is enough to check property $(P)$ on domains $\Omega$ with non-compact closure.

Proposition 2.9. Let $\varphi, T$ satisfy $(\varphi 1),(\varphi 3),(\varphi 4),(\varphi 5)$ and $(T 1), X \in \mathfrak{X}(M)$ and define $Q$ as in (8). Then the equivalent conditions in Theorem 2.2 are also equivalent to the following:

v) $Q$ satisfies $(P)$ for some admissible pair $(c, h)$ with $c \in L^{\infty}(M)$, where $\Omega$ in $(P)$ has non-compact closure.

We close this paragraph with an application of the weak maximum principle to prove a comparison result for subsolutions and supersolutions of semilinear differential equations.

Theorem 2.10. Let $Q$ be the linear differential operator defined in (9) and let $u, v \in C^{2}(M)$ satisfy

$$
u, v \in L^{\infty}(M) \cap C^{2}(M), \quad u \geq 0, \quad \inf _{M} v>0
$$

and

$$
Q u \geq f(x, u), \quad Q v \leq f(x, v) \quad \text { on } M,
$$

where $f: M \times \mathbb{R}_{0}^{+} \rightarrow \mathbb{R}$ is locally Lipschitz in the variable s, uniformly in $x \in M$ and such that

$$
\forall s_{1}, s_{2}, \quad 0<s_{1}<s_{2}, \quad \inf _{x \in M}\left(\frac{f\left(x, s_{2}\right)}{s_{2}}-\frac{f\left(x, s_{1}\right)}{s_{1}}\right)>0 .
$$

If the WMP holds for $Q$ on $M$, then $u \leq v$ on $M$. 
The above comparison result is very general and can be applied to a wide variety of differential equations. As an example, we consider the Lichnerowicztype equation

$$
Q u+a(x) u-b(x) u^{\sigma}+c(x) u^{\tau}=0,
$$

where $Q$ is as in (9) and linear (that is, $\varphi(x, s)=s$ ), $a(x), b(x), c(x)$ are continuous, $\sigma>1$ and $\tau<1$.

We note that, for $f(x, s)=-a(x) s+b(x) s^{\sigma}-c(x) s^{\tau}$, the assumptions of the theorem are satisfied provided $a(x), b(x), c(x)$ are bounded, $b(x) \geq 0, c(x) \geq 0$ and either $\inf _{M} b>0$ or $\inf _{M} c>0$. In particular, the assumption on the local Lipschitz continuity of the function $f$ in the variable $s$ is satisfied even though $\tau<1$ since, as it will be apparent from the proof, this assumption is only necessary for $s$ in the range of the subsolution and supersolution, which, for the Lichnerowicz equation, is assumed bounded below by a positive constant.

\section{Proofs of the analytic results}

The proof of Theorem 2.2 is quite straightforward once we recall the validity of the next result (see Theorem 4.6 of [2], or [3]).

Theorem 3.1. The WMP holds for $Q$ if and only if the open weak maximum principle, for short $O W M P$, holds for $Q$ on $M$, that is, for each $g \in C^{0}(\mathbb{R})$, for each open set $\Omega \subset M$ with $\partial \Omega \neq \varnothing$, each solution $v \in C^{0}(\bar{\Omega}) \cap C^{1}(\Omega)$ of

$$
\left\{\begin{array}{l}
Q v \geq g(v) \quad \text { on } \Omega \\
\sup _{\Omega} v<+\infty
\end{array}\right.
$$

satisfies either

$$
\sup _{\Omega} v=\sup _{\partial \Omega} v
$$

or

$$
g\left(\sup _{\Omega} v\right) \leq 0 .
$$

We are now ready for the

Proof (of Theorem 2.2). We prove the chain of implications $i) \Rightarrow i i) \Rightarrow i i i) \Rightarrow$ $i v) \Rightarrow i)$.

$i) \Rightarrow i i)$. Suppose that $Q$ satisfies the WMP and choose an admissible pair $(c, h)$ satisfying

$$
c_{-} \in L^{\infty}(M), \quad \operatorname{sh}(s) \geq 0 \text { on } \mathbb{R}
$$

and a function $0<\beta \in C^{0}(\mathbb{R})$. Let $\Omega \subset M$ be an open set with $\partial \Omega \neq \varnothing$ and $v \in C^{0}(\bar{\Omega}) \cap C^{1}(\Omega)$ be a solution of (12). From the differential inequality in 
(12) and since $v$ and $h(v)$ have the same sign, we deduce

$$
Q v \geq \beta(v)+c_{-}(x) h(v) \geq \beta(v)+\left\|c_{-}\right\|_{L^{\infty}(M)} h(v)
$$

By Theorem 3.1 applied with the choice $g(s)=\beta(s)+\left\|c_{-}\right\|_{L^{\infty}(M)} h(s)$, we deduce that either

$$
\sup _{\Omega} v=\sup _{\partial \Omega} v<0
$$

or

$$
g\left(\sup _{\Omega} v\right) \leq 0
$$

In the latter case, since $\beta>0$ and $h(0)=0$ imply $g(0)=\beta(0)>0$, necessarily

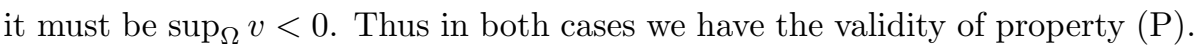

$i i) \Rightarrow$ iii). Let $v$ satisfy (12) for an admissible pair $(c, h)$ with $c \in L^{\infty}(M)$

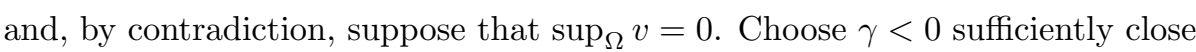
to zero in such a way that

$$
\|c\|_{L^{\infty}(M)}\|h\|_{L^{\infty}([\gamma, 0])}<\inf _{[\gamma, 0]} \beta .
$$

This is possible since $\beta, h \in C^{0}(\mathbb{R}), \beta>0$ and $h(0)=0$. Since $v$ cannot be constant, we can also choose $\gamma$ in such a way that the open set

$$
\Omega_{\gamma}=\{x \in \Omega: v(x)>\gamma\}
$$

satisfies $\bar{\Omega}_{\gamma} \subset \Omega$. In particular we have

$$
\left\{\begin{array}{l}
Q v \geq \beta(v)-c(x) h(v) \geq \inf _{[\gamma, 0]} \beta-\|c\|_{L^{\infty}(M)}\|h\|_{L^{\infty}([\gamma, 0])}=\bar{\beta}>0 \quad \text { on } \Omega_{\gamma} \\
\sup _{\partial \Omega_{\gamma}} v=\gamma<0 .
\end{array}\right.
$$

In other words

$$
\left\{\begin{array}{l}
Q v \geq \bar{\beta}>0 \quad \text { on } \Omega_{\gamma} \\
\sup _{\partial \Omega_{\gamma}} v \gamma<0 .
\end{array}\right.
$$

Thus, by $i i)$ applied on $\Omega_{\gamma}$ with the pair $(0,0)$, which is admissible and satisfies (17), we have $\sup v<0$, a contradiction.

$i i i) \Rightarrow i v)$ is obvious.

$i v) \Rightarrow i)$. Consider the admissible pair $(c, h)$ granted by $i v)$ with $c \in L^{\infty}(M)$. By contradiction, let $u \in C^{1}(M)$ be such that $u^{*}=\sup _{M} u<+\infty$ and let $\gamma<u^{*}$, 
$\Lambda>0$ be such that $Q u \geq \Lambda$ on

$$
\Omega_{\gamma}=\{x \in M: u(x)>\gamma\}
$$

Using the fact that $Q(u+c)=Q(u)$ for each constant $c$, possibly adding a constant to $u$ we can suppose that $\gamma>0$ and possibly increasing $\gamma$ we can ensure that $\partial \Omega_{\gamma} \neq \varnothing$ and furthermore that

$$
\|c\|_{L^{\infty}(M)}\|h\|_{L^{\infty}\left(\left[\gamma-u^{*}, 0\right]\right)}<\Lambda .
$$

On $\bar{\Omega}_{\gamma}$ we define $v=u-u^{*}$. For $x \in \Omega_{\gamma}, v(x) \in\left[\gamma-u^{*}, 0\right]$, hence we have

$$
\begin{aligned}
Q v+c(x) h(v) & \geq Q v-\|c\|_{L^{\infty}(M)}\|h\|_{L^{\infty}\left(\left[\gamma-u^{*}, 0\right]\right)} \\
& \geq \Lambda-\|c\|_{L^{\infty}(M)}\|h\|_{L^{\infty}\left(\left[\gamma-u^{*}, 0\right]\right)}=\beta>0 .
\end{aligned}
$$

By property (P) for the admissible pair $(c, h)$ applied to $v$ on $\Omega_{\gamma}$ and with the choice of $\beta(s)=\beta>0$ defined above we have $\sup _{\Omega_{\gamma}} v<0$, a contradiction.

We now turn to the proof of the comparison principle stated in Proposition 2.7. To this end, we let $Q_{0}$ be as defined in (14). The next lemma provides the necessary monotonicity condition on $Q_{0}$ for the validity of a comparison result.

First let $\varphi$ satisfy $(\varphi 1),(\varphi 3),(\varphi 4)$ and, for $x \in M$ and $\xi \in T_{x} M$ fixed, define the function

$$
g_{x, \xi}: T_{x} M \backslash\{0\} \longrightarrow \mathbb{R}
$$

by setting

$$
g_{x, \xi}(\eta)=T\left(|\eta|^{-1} \varphi(x,|\eta|) \eta, \xi\right) .
$$

Since

$$
\left|g_{x, \xi}(\eta)\right| \leq|T|_{x} \varphi(x,|\eta|)|\xi|,
$$

the validity of $(\varphi 1)$ implies that

$$
g_{x, \xi}(\eta) \rightarrow 0 \text { as } \eta \rightarrow 0 \text {. }
$$

This allows us to extend $g_{x, \xi}: T_{x} M \rightarrow \mathbb{R}$ continuously by setting $g_{x, \xi}(0)=0$.

Lemma 3.2. Assume the validity of $(\varphi 1),(\varphi 3),(\varphi 4)$ and $(\varphi 5)$ and define $g_{x, \xi}$ for $x \in M, \xi \in T_{x} M$ as above. Let $\nabla u, \nabla v \in T_{x} M$ and set $X_{t}=t \nabla u+(1-t) \nabla v$ for $t \in[0,1]$. Suppose that $|\nabla u|+|\nabla v|>0$ and let $T$ be a 2-covariant tensor 
field on $M$. Then at $x$ we have

$$
\begin{aligned}
& \bar{g}(x)=g_{x, \nabla u-\nabla v}(\nabla u)-g_{x, \nabla u-\nabla v}(\nabla v)= \\
& =\int_{0}^{1}\left\{\frac{\varphi\left(x,\left|X_{t}\right|\right)}{\left|X_{t}\right|} T(\nabla u-\nabla v, \nabla u-\nabla v)+\right. \\
& \left.\frac{1}{\left|X_{t}\right|^{2}}\left[\frac{\partial \varphi}{\partial s}\left(x,\left|X_{t}\right|\right)-\frac{\varphi\left(x,\left|X_{t}\right|\right)}{\left|X_{t}\right|}\right]\left\langle X_{t}, \nabla u-\nabla v\right\rangle T\left(X_{t}, \nabla u-\nabla v\right)\right\} d t .
\end{aligned}
$$

Furthermore, if $(T 1)$ holds, then $\bar{g}(x) \geq 0$ and $\bar{g}(x)=0$ if and only if $\nabla u=\nabla v$.

Proof. First assume that $X_{t} \neq 0$ on $[0,1]$. Let $\gamma:[0,1] \rightarrow M$ be the constant curve $\gamma(t)=x$ for $t \in[0,1]$ and consider $X_{t}$ as a vector field along $\gamma$. To simplify notations, we set $Y=\nabla u-\nabla v$. Let $\left\{e_{i}\right\}$ be a local orthonormal frame around $x$ satisfying $\left(\nabla_{e_{j}} e_{i}\right)(x)=0$ for all $i, j=1, \ldots, m$. Using the latter, jointly with the properties of covariant differentiation $\frac{D}{d t}$ along a curve, the fact that $\dot{\gamma} \equiv 0$ on $[0,1]$ and $X_{t} \neq 0$ on $[0,1]$, we have

$$
\begin{aligned}
& \frac{d}{d t}\left\langle\left|X_{t}\right|^{-1} \varphi\left(x,\left|X_{t}\right|\right) T\left(X_{t}, \quad\right)^{\sharp}, Y\right\rangle=\left\langle\frac{D}{d t}\left(\left|X_{t}\right|^{-1} \varphi\left(x,\left|X_{t}\right|\right) T\left(X_{t}, \quad\right)^{\sharp}\right), Y\right\rangle= \\
& =\left\langle-\left|X_{t}\right|^{-3}\left\langle\frac{D}{d t} X_{t}, X_{t}\right\rangle \varphi\left(x,\left|X_{t}\right|\right) T\left(X_{t}, \quad\right)^{\sharp}, Y\right\rangle+ \\
& +\left\langle\left|X_{t}\right|^{-2} \frac{\partial \varphi}{\partial s}\left(x,\left|X_{t}\right|\right)\left\langle\frac{D}{d t} X_{t}, X_{t}\right\rangle T\left(X_{t}, \quad\right)^{\sharp}+\left|X_{t}\right|^{-1} \varphi\left(x,\left|X_{t}\right|\right) \frac{D}{d t} T\left(X_{t}, \quad\right)^{\sharp}, Y\right\rangle= \\
& =-\left|X_{t}\right|^{-3} \varphi\left(x,\left|X_{t}\right|\right)\left\langle X_{t}, Y\right\rangle T\left(X_{t}, Y\right)+\left|X_{t}\right|^{-2} \frac{\partial \varphi}{\partial s}\left(x,\left|X_{t}\right|\right)\left\langle X_{t}, Y\right\rangle T\left(X_{t}, Y\right)+ \\
& +\left|X_{t}\right|^{-1} \varphi\left(x,\left|X_{t}\right|\right)\left\langle\frac{D}{d t}\left(t T(\nabla u,)^{\sharp}+(1-t) T(\nabla v, \quad)^{\sharp}\right), Y\right\rangle= \\
& =\frac{\varphi\left(x,\left|X_{t}\right|\right)}{\left|X_{t}\right|} T(Y, Y)+\frac{1}{\left|X_{t}\right|^{2}}\left[\frac{\partial \varphi}{\partial s}\left(x,\left|X_{t}\right|\right)-\frac{\varphi\left(x,\left|X_{t}\right|\right)}{\left|X_{t}\right|}\right]\left\langle X_{t}, Y\right\rangle T\left(X_{t}, Y\right) .
\end{aligned}
$$

Thus in this case the result follows immediately by integration. Now suppose there exists $t_{0} \in[0,1]$ with $X_{t_{0}}=0$. Then, $X_{t}=\left(t-t_{0}\right) Y$ and necessarily $Y \neq 0$ (otherwise, $\nabla u=\nabla v=0$ ), whence $t_{0}$ is unique. The cases $t_{0}=0, t_{0}=1$ are simpler, so let us assume $t_{0} \in(0,1)$. Let $I$ be the integrand in (18). For $\varepsilon>0$ sufficiently small, integrating (19) on the intervals $\left[0, t_{0}-\varepsilon\right]$ and $\left[t_{0}+\varepsilon, 1\right]$, we get

$$
\begin{aligned}
\int_{0}^{t_{0}-\varepsilon} I+\int_{t_{0}+\varepsilon}^{1} I= & g_{x, \nabla u-\nabla v}\left(X_{t_{0}-\varepsilon}\right)-g_{x, \nabla u-\nabla v}(\nabla v) \\
& +g_{x, \nabla u-\nabla v}(\nabla u)-g_{x, \nabla u-\nabla v}\left(X_{t_{0}+\varepsilon}\right) .
\end{aligned}
$$

By the continuity of $g_{x, \xi}$, its linearity in $\xi$ and since $X_{t_{0}}=0$, the RHS of the above converges to $\bar{g}(x)$ as $\varepsilon \rightarrow 0^{+}$. On the other hand, because of $(\varphi 1)$ and 
$(\varphi 4)$ we get

$$
\frac{\partial \varphi}{\partial s}(x, s) \in L^{1}\left(0^{+}\right)
$$

Coupling with $(\varphi 5)$ and using $X_{t}=\left(t-t_{0}\right) Y$, the LHS converges to $\int_{0}^{1} I$. Under the validity of $(T 1)$, the fact that $\bar{g}(x) \geq 0$ and $\bar{g}(x)=0$ if and only if $\nabla u=\nabla v$ follows immediately from (18).

Proof (of Proposition 2.7). Suppose by contradiction that, for some $\varepsilon>0$, the relatively compact open set $\Omega_{\varepsilon}=\{x \in \Omega: u(x)>v(x)+\varepsilon\}$ is nonempty. Note that $\bar{\Omega}_{\varepsilon} \subset \Omega$. Let $\alpha \in C^{1}(\mathbb{R})$ satisfy

$$
\alpha \equiv 0 \text { on }(-\infty, \varepsilon], \quad \alpha^{\prime}>0 \text { on }(\varepsilon,+\infty)
$$

We test the differential inequality in (15) with the test function $w=\alpha(u-v) \in$ $C_{c}^{1}(\Omega)$ to get

$$
\int_{\Omega_{\varepsilon}} \alpha^{\prime}(u-v) T\left(|\nabla u|^{-1} \varphi(x,|\nabla u|) \nabla u-|\nabla v|^{-1} \varphi(x,|\nabla v|) \nabla v, \nabla u-\nabla v\right) \leq 0 .
$$

However, by Lemma 3.2,

$$
\bar{g}(x)=T\left(|\nabla u|^{-1} \varphi(x,|\nabla u|) \nabla u-|\nabla v|^{-1} \varphi(x,|\nabla v|) \nabla v, \nabla u-\nabla v\right) \geq 0 .
$$

Since $\alpha^{\prime}(u-v)>0$ on $\Omega_{\varepsilon}$, it follows that $\bar{g} \equiv 0$ on $\Omega_{\varepsilon}$. Again by Lemma 3.2, $\nabla u \equiv \nabla v$ on $\Omega_{\varepsilon} \cap\{|\nabla u|+|\nabla v|>0\}$, hence on $\Omega_{\varepsilon}$. Integrating, $u-v$ is constant on each connected component of $\Omega_{\varepsilon}$, contradicting its very definition.

With this preparation we are ready to prove Proposition 2.9.

Proof (of Proposition 2.9). Since item $i v$ ) in Theorem 2.2 implies item $v$ ) in Proposition 2.9, it is enough to prove that, in our assumptions, $(\mathrm{P})$ automatically holds for $(c, h)$ if $\Omega$ has compact closure. Therefore, pick a solution $v \in C^{0}(\bar{\Omega}) \cap$ $C^{1}(\Omega)$ of

$$
\begin{cases}Q v+c(x) h(v) \geq \beta(v) & \text { on } \Omega \subset \subset M \\ v \leq 0 & \text { on } \Omega \\ \sup _{\partial \Omega} v<0, & \end{cases}
$$

and by contradiction suppose that $\sup _{\Omega} v=0$. Then, by compactness, 0 is attained at an interior point of $\bar{\Omega}$ that is, at a point of $\Omega$. Define the compact set $\Gamma=\{x \in \Omega: v(x)=0\}$. Fix $\gamma<0$ sufficiently close to zero in order that

$$
\Omega_{\gamma}=\{x \in \Omega: v(x)>\gamma\} \subset \subset \Omega
$$


On $\Omega_{\gamma}$ we have

$$
\begin{aligned}
Q_{0} v & =Q v+\langle X, \nabla v\rangle \geq \beta(v)-c(x) h(v)+\langle X, \nabla v\rangle \\
& \geq \beta(v)-\|X\|_{L^{\infty}(\Omega)}\|\nabla v\|_{L^{\infty}\left(\Omega_{\gamma}\right)}-\|c\|_{L^{\infty}(M)}\|h\|_{L^{\infty}([\gamma, 0])} \\
& \geq \inf _{[\gamma, 0]} \beta-\|X\|_{L^{\infty}(\Omega)}\|\nabla v\|_{L^{\infty}\left(\Omega_{\gamma}\right)}-\|c\|_{L^{\infty}(M)}\|h\|_{L^{\infty}([\gamma, 0])} .
\end{aligned}
$$

Since $\Omega_{\gamma}$ shrinks to $\Gamma$ as $\gamma \uparrow 0$ and $v \in C^{1}(\Omega)$ satisfies $\nabla v=0$ on $\Gamma$, we can make $\|\nabla v\|_{L^{\infty}\left(\Omega_{\gamma}\right)}$ as small as we wish up to choosing $\gamma$ close enough to zero. The same happens for $\|h\|_{L^{\infty}([\gamma, 0])}$ since $h(0)=0$ and $h$ is continuous. Since $0<\beta \in C^{0}(\mathbb{R})$, from (20) we deduce that

$$
Q_{0} v \geq \frac{1}{2} \beta(0)>0 \quad \text { on } \Omega_{\gamma}
$$

if $\gamma$ is close enough to zero. Since the constant $\gamma$ satisfies $Q_{0} \gamma=0$ and $v=\gamma$ on $\partial \Omega_{\gamma}$, by Proposition 2.7 we deduce that $v \leq \gamma$ on $\Omega_{\gamma}$; contradiction.

We now come to the

Proof (of Theorem 2.6). Let $(c, h)$ be an admissible pair as in $(B K)$ with

$$
c_{-} \in L^{\infty}(M), \quad \operatorname{sh}(s) \geq 0 \text { on } \mathbb{R} .
$$

By contradiction suppose the existence of an open set $\Omega \subset M$ with $\partial \Omega \neq \varnothing$, of $0<\beta \in C^{0}(\mathbb{R})$ and $v \in C^{0}(\bar{\Omega}) \cap C^{1}(\Omega)$ solution of

$$
\begin{cases}Q v+c(x) h(v) \geq \beta(v) & \text { on } \Omega \\ v \leq 0 & \text { on } \Omega \\ \sup _{\partial \Omega} v<0, & \end{cases}
$$

for which $\sup _{\Omega} v=0$. Set $\beta_{0}=\inf _{[-1,0]} \beta>0$ and choose $\varepsilon$ and $\sigma$ satisfying

$$
\varepsilon \in\left(0, \frac{\beta_{0}}{8}\right), \quad 0<\sigma<\min \left\{B_{1}, B_{2}, \frac{1}{2},-\sup _{\partial \Omega} v\right\}
$$

where $B_{j}=B_{j}(\varepsilon)$ are as in $(B K)$. Furthermore, we require $\sigma$ to be small enough that if $s \in\left[-B_{1}, B_{1}\right]$ and $|\bar{s}|<2 \sigma$, then

$$
|h(s+\bar{s})-h(s)| \leq \frac{\beta_{0}}{4\left\|c_{-}\right\|_{L^{\infty}(M)}},
$$

with the agreement that this is automatically satisfied if $c_{-} \equiv 0$. Note that $(23)$ is possible since $h(s) \in C^{0}(\mathbb{R})$. 
Since $\sup _{\Omega} v=0$, the set

$$
\Omega_{-\sigma}=\{x \in \Omega: v(x)>-\sigma\}
$$

is not empty and we choose $x_{0} \in \Omega_{-\sigma}$. Next we consider the function $\gamma=\gamma_{x_{0}, \varepsilon}$ granted by $(B K)$ and we let $a>0$ sufficiently small to satisfy $\sigma<B_{2}-a$. Because of property $i$ ) in Definition 2.4,

$$
x_{0} \in U=\left\{x \in M: \gamma_{x_{0}, \varepsilon}(x)<B_{2}-a\right\} .
$$

By property $i v$ ) of Definition 2.4, $U$ is relatively compact by the Hopf-Rinow theorem, since $M$ is complete. Set

$$
w(x)=v(x)-\gamma_{x_{0}, \varepsilon}(x) \quad \text { on } \bar{\Omega} .
$$

By properties $i$ ) and $i i$ ) of Definition 2.4, (22), the fact that $x_{0} \in \Omega_{-\sigma}$ and the inequalities $\sigma<B_{2}-a, v \leq 0$ on $\bar{\Omega}$, we deduce

$$
\begin{aligned}
& \text { i) } \quad w \leq 0 \quad \text { on } \Omega \text {, } \\
& \text { ii) } \quad w(x)=v(x)-\gamma_{x_{0}, \varepsilon}(x) \leq-\gamma_{x_{0}, \varepsilon}(x)<v\left(x_{0}\right) \\
& =v\left(x_{0}\right)-\gamma_{x_{0}, \varepsilon}\left(x_{0}\right)=w\left(x_{0}\right) \quad \text { on } \partial U \cap \bar{\Omega} \text {. }
\end{aligned}
$$

Let $w^{*} \leq 0$ denote the maximum of $w$ on the compact set $\overline{U \cap \Omega}$ and let $x_{1}$ be a maximum point that, by (24) ii), lies in $U \cap \bar{\Omega}$. Using $(22), v\left(x_{0}\right)>-\sigma$ and $i$ ) of Definition 2.4, we have

$$
0 \geq w^{*}=w\left(x_{1}\right) \geq w\left(x_{0}\right)=v\left(x_{0}\right)>-\sigma>\sup _{\partial \Omega} v \geq \sup _{\partial \Omega} w
$$

so that $x_{1} \notin \partial \Omega$. It follows that

$$
x_{1} \in U \cap \Omega \text {. }
$$

Now consider the function

$$
u(x)=\gamma_{x_{0}, \varepsilon}(x)+w^{*} \quad \text { on } \bar{\Omega} .
$$

Note that, on $\overline{U \cap \Omega}, u-v=w^{*}-w$, hence

$$
\text { i) } u-v \geq 0 \quad \text { on } \overline{U \cap \Omega}, \quad \text { ii) }(u-v)\left(x_{1}\right)=0
$$


and by (25) and (24)

$$
u-v=w^{*}-w>0 \quad \text { on } \partial \Omega \cup(\partial U \cap \bar{\Omega}) \supset \partial(U \cap \Omega) .
$$

Fix $\bar{\varepsilon}>0$ and let

$$
\Omega_{\bar{\varepsilon}}=\{x \in \overline{U \cap \Omega}: u(x)-v(x)<\bar{\varepsilon}\} .
$$

In view of (26) and (27), $x_{1} \in \Omega_{\bar{\varepsilon}}$ for each $\bar{\varepsilon}>0$; furthermore, for $\bar{\varepsilon}$ sufficiently small, say $\bar{\varepsilon} \leq \varepsilon_{0}$ for some fixed $\varepsilon_{0}, \bar{\Omega}_{\bar{\varepsilon}} \subset U \cap \Omega$, which follows immediately from (27).

As $\bar{\varepsilon} \downarrow 0^{+}, \Omega_{\bar{\varepsilon}}$ shrinks to the compact set

$$
\{x \in U \cap \Omega: u(x)=v(x)\},
$$

where, by (26), $\nabla u=\nabla v$. Taking into account that $u, v \in C^{1}\left(\Omega_{\bar{\varepsilon}}\right)$, up to choosing $\bar{\varepsilon}$ small enough we can guarantee that

$$
|\nabla u-\nabla v|<\frac{\beta_{0}}{4\|X\|_{L^{\infty}\left(\Omega_{\varepsilon_{0}}\right)}} \quad \text { on } \Omega_{\bar{\varepsilon}},
$$

where $X$ is the vector field in the definition of $Q$, and we agree that (28) is automatically satisfied if $X \equiv 0$. Up to shrinking $\bar{\varepsilon}$ further, we can also assume that

$$
0<\bar{\varepsilon}<\min \left\{\frac{1}{2}, \sigma\right\} .
$$

Define

$$
\bar{u}(x)=u(x)-\bar{\varepsilon}=\gamma_{x_{0}, \varepsilon}(x)+w^{*}-\bar{\varepsilon}
$$

on $\bar{\Omega}_{\bar{\varepsilon}}$ and observe that, by the definition of $\Omega_{\bar{\varepsilon}}$ and by (28) we have

$$
\text { i) } \Omega_{\bar{\varepsilon}}=\{x \in \Omega \cap U: \bar{u}(x)<v(x)\} ; \quad \text { ii) }|\nabla \bar{u}-\nabla v|<\frac{\beta_{0}}{4\|X\|_{L^{\infty}\left(\Omega_{\varepsilon_{0}}\right)}} \quad \text { on } \Omega_{\bar{\varepsilon}} \text {. }
$$

Now because of $i i i$ ) of Definition 2.4 and the definition of $Q$,

$$
Q \bar{u}=Q\left(w^{*}-\bar{\varepsilon}+\gamma_{x_{0}, \varepsilon}\right)=Q \gamma_{x_{0}, \varepsilon} \leq \varepsilon-c(x) h\left(\gamma_{x_{0}, \varepsilon}\right)
$$

on $\Omega_{\bar{\varepsilon}}$. Since $\gamma_{x_{0}, \varepsilon} \geq 0$ on $M, \bar{u}=u-\bar{\varepsilon} \leq v \leq 0$ on $\Omega_{\bar{\varepsilon}}$ and $h(s)$ has the same sign of $s$, we infer that

$$
h\left(\gamma_{x_{0}, \varepsilon}\right) \geq 0, \quad h(\bar{u}) \leq 0 \quad \text { on } \Omega_{\bar{\varepsilon}} .
$$


Hence, from (30) we deduce that on $\Omega_{\bar{\varepsilon}}$

$$
\begin{aligned}
Q \bar{u} & \leq \varepsilon+c_{-}(x) h\left(\gamma_{x_{0}, \varepsilon}\right)=\varepsilon+c_{-}(x)\left\{h\left(\gamma_{x_{0}, \varepsilon}\right)-h\left(\gamma_{x_{0}, \varepsilon}+w^{*}-\bar{\varepsilon}\right)\right\}+c_{-}(x) h(\bar{u}) \\
& \leq \varepsilon+\left\|c_{-}\right\|_{L^{\infty}(M)}\left|h\left(\gamma_{x_{0}, \varepsilon}\right)-h\left(\gamma_{x_{0}, \varepsilon}+w^{*}-\bar{\varepsilon}\right)\right| .
\end{aligned}
$$

From $i$ ) in Definition 2.4 we deduce that $0 \leq \gamma_{x_{0}, \varepsilon} \leq B_{1}$, and coupling (25) and (29) we get $\left|w^{*}-\bar{\varepsilon}\right|<2 \sigma$. Using (23), we therefore obtain

$$
\left|h\left(\gamma_{x_{0}, \varepsilon}\right)-h\left(\gamma_{x_{0}, \varepsilon}+w^{*}-\bar{\varepsilon}\right)\right|<\frac{\beta_{0}}{4\left\|c_{-}\right\|_{L^{\infty}(M)}} .
$$

Inserting into (31) we obtain

$$
Q \bar{u} \leq \varepsilon+\frac{\beta_{0}}{4} \quad \text { on } \Omega_{\bar{\varepsilon}}
$$

On the other hand, on $\Omega_{\bar{\varepsilon}}$,

$$
0 \geq v>\bar{u}=u-\bar{\varepsilon}=\gamma_{x_{0}, \varepsilon}+w^{*}-\bar{\varepsilon} \geq w^{*}-\bar{\varepsilon} \geq-2 \sigma>-1
$$

Hence, by the definition of $\beta_{0}$,

$$
\beta(v) \geq \beta_{0} \quad \text { on } \Omega_{\bar{\varepsilon}}
$$

Using (21) together with $|v|<2 \sigma, h(0)=0$ and (23) again, we get on $\Omega_{\bar{\varepsilon}}$

$$
Q v \geq \beta_{0}-c(x) h(v) \geq \beta_{0}-\left\|c_{-}\right\|_{L^{\infty}(M)}|h(v)| \geq \frac{3}{4} \beta_{0} .
$$

Putting together (22), (32), (33) and (28) on $\Omega_{\bar{\varepsilon}}=\{x \in U \cap \Omega: v(x)>u(x)\}$ we get

$$
\begin{aligned}
Q_{0} v-Q_{0} \bar{u} & =Q v-Q \bar{u}-\langle X, \nabla v-\nabla \bar{u}\rangle \geq \\
& \geq \frac{3}{4} \beta_{0}-\varepsilon-\frac{\beta_{0}}{4}-\|X\|_{L^{\infty}\left(\Omega_{\varepsilon_{0}}\right)}\|\nabla v-\nabla \bar{u}\|_{L^{\infty}\left(\Omega_{\bar{\varepsilon}}\right)} \\
& \geq \frac{\beta_{0}}{8}
\end{aligned}
$$

and $v=\bar{u}$ on $\partial \Omega_{\bar{\varepsilon}}$. In our assumptions we are able to use the comparison result in Proposition 2.7 on $\Omega_{\bar{\varepsilon}}$ to deduce that $v \leq \bar{u}$ on $\Omega_{\bar{\varepsilon}}$, contradiction. This completes the proof of Theorem 2.6.

We now turn to Theorem 2.10. The proof follows [5, Lemma 3.4], but we reproduce it here for the sake of completeness. To this end, we state the following 
Lemma 3.3. Let $u_{1}, u_{2} \in C(M)$ be two positive, bounded functions satisfying

$$
\inf _{M} u_{1}>0, \quad \inf _{M}\left(u_{2}-u_{1}\right)>0 .
$$

If $f$ is locally Lipschitz continuous in the variable $s \in \mathbb{R}_{0}^{+}$, uniformly in $x \in M$, and (16) holds, then there exists $\varepsilon>0$ such that

$$
\forall x \in M, \quad \frac{u_{2}(x)}{u_{1}(x)} f\left(x, u_{1}(x)\right) \leq f\left(x, u_{2}(x)\right)-\varepsilon .
$$

The proof of this lemma is elementary and can be found in [5].

The next proof is similar, in spirit, to that of Theorem 4.3 of [1] or that of Theorem 3.1 in [24]. However, the new form of the WMP enables us to apply directly the argument in [5].

Proof (of Theorem 2.10). Since $\inf _{M} v>0$, there exists $k>0$ such that $k v \geq u$ on $M$. Set

$$
k^{*}=\inf \{k>0: k v-u \geq 0 \text { on } M\}
$$

and suppose $k^{*}>1$. The function $w=u-k^{*} v$ is nonpositive, of class $C^{2}$ and, by the linearity of $Q$, it satisfies

$$
Q w \geq f(x, u)-k^{*} f(x, v)
$$

on $M$. Since

$$
\inf _{M}\left(k^{*} v-v\right)=\left(k^{*}-1\right) \inf v>0,
$$

we can apply Lemma 3.3 to the functions $u_{1}=v$ and $u_{2}=k^{*} v$, so there exists $\varepsilon>0$ such that

$$
\frac{k^{*} v}{v} f(x, v) \leq f\left(x, k^{*} v\right)-\varepsilon,
$$

which yields

$$
Q w \geq f(x, u)-f\left(x, k^{*} v\right)+\varepsilon \quad \text { on } M
$$

Setting

$$
c(x)= \begin{cases}\frac{f(x, u(x))-f\left(x, k^{*} v(x)\right)}{u(x)-k^{*} v(x)} & \text { if } k^{*} v(x) \neq u(x) \\ 0 & \text { if } k^{*} v(x)=u(x),\end{cases}
$$

we see that $c \in L^{\infty}(M)$ since $f(x, \cdot)$ is locally Lipschitz. Moreover, $w$ satisfies

$$
Q w-c(x) w \geq \varepsilon
$$

We can therefore apply the WMP to $w$ and deduce that $\sup _{M} w<0$. Thus we can choose $\delta>0$ so small that $w+\delta v \leq 0$ on $M$, implying, by the definition of 
$w$, that $\left(k^{*}-\delta\right) v \geq u$, contradicting the very definition of $k^{*}$.

Remark 3.4. As already pointed out, if also $\inf _{M} u>0$, then we can relax the Lipschitz assumption to

$f: M \times \mathbb{R}^{+} \rightarrow \mathbb{R}$ is locally Lipschitz in the variable $s \in \mathbb{R}^{+}$, uniformly in $x \in M$, that is, $f$ is not required to be Lipschitz in a neighbourhood of $s=0$.

From Theorem 2.10 we immediately obtain the validity of the next

Corollary 3.5. Let $Q$ be the linear differential operator defined in (9). Let $f: M \times \mathbb{R}^{+} \rightarrow \mathbb{R}$ be locally Lipschitz in the variable $s$, uniformly in $x \in M$ and such that (16) holds. Assume the validity of the WMP for $Q$ on $M$. Then the equation

$$
Q u=f(x, u)
$$

has at most one $C^{2}$ solution $u$ satisfying

$$
C^{-1} \leq u \leq C \quad \text { on } M
$$

for some constant $C>0$.

Now Theorem 1.1 follows directly from the above Corollary. We conclude with a remark.

Remark 3.6. We first stress that the lower bound $u \geq C^{-1}$ in (3) is indeed necessary for the validity of the Theorem. Indeed, suppose $c(x)=0$ and consider the Yamabe equation

$$
\Delta u+\frac{m(m-2)}{2} u-u^{\frac{m+2}{m-2}}=0, \quad m \geq 3
$$

on $\mathbb{H}^{m}$, the $m$-dimensional hyperbolic space of constant sectional curvature -1 . Here the assumptions of Theorem 1.1 are satisfied, but as shown in Section 5.2 of [15], having fixed an origin $o \in \mathbb{H}^{m}$ and set $r(x)=\operatorname{dist}(x, o)$, the family of distinct functions

$$
u_{a}(x)=\frac{1}{m(m-2) a^{2}}\left(a^{2}-\tanh ^{2} \frac{r(x)}{2}\right)^{-\frac{m-2}{2}}\left[2 \cosh ^{2} \frac{r(x)}{2}\right]^{-\frac{m-2}{2}} \quad a>1
$$

is a family of solutions of (34) satisfying

$$
u_{a}(x) \rightarrow 0 \quad \text { as } r(x) \rightarrow \infty .
$$




\section{References}

[1] G. Albanese and M. Rigoli, Lichnerowicz-type equations on complete manifolds, Adv. Nonlinear Anal. 5 (2016), no. 3, 223-250.

[2] L. J. Alías, P. Mastrolia, and M. Rigoli, Maximum principles and geometric applications, Springer Monographs in Mathematics, Springer, Cham, 2016.

[3] L. J. Alías, J. F. R. Miranda, and M. Rigoli, A new open form of the weak maximum principle and geometric applications, Comm. Anal. Geom. 24 (2016), no. 1, 1-43.

[4] P. Antonini, D. Mugnai, and P. Pucci, Quasilinear elliptic inequalities on complete Riemannian manifolds, J. Math. Pures Appl. (9) 87 (2007), no. 6 , $582-600$.

[5] H. Berestycki, F. Hamel, and L. Rossi, Liouville-type results for semilinear elliptic equations in unbounded domains, Ann. Mat. Pura Appl. (4) 186 (2007), no. 3, 469-507.

[6] Y. Choquet-Bruhat, J. Isenberg, and D. Pollack, The constraint equations for the Einstein-scalar field system on compact manifolds, Classical Quantum Gravity 24 (2007), no. 4, 809-828.

[7] Y. Choquet-Bruhat and J. York, The Cauchy problem, General Relativity (A. Held, ed.), Plenum Press, New York (1980), 99-172.

[8] T. Colding and W. Minicozzi, Generic mean curvature flow I: generic singularities, Ann. of Math. 175 (2012), no. 2, 755-833.

[9] M. Fernández-López and E. García-Río, Maximum principles and gradient Ricci solitons, J. Differential Equations 251 (2011), no. 1, 73-81.

[10] D. Gilbarg and N. S. Trudinger, Elliptic partial differential equations of second order, Classics in Mathematics, Springer-Verlag, Berlin, 2001, Reprint of the 1998 edition.

[11] A. Lichnerowicz, L'intégration des équations de la gravitation relativiste et le problème des $n$ corps, J. Math. Pures Appl. (9) 23 (1944), 37-63.

[12] C. Mantegazza, Lecture notes on mean curvature flow, Progress in Mathematics, vol. 290, Birkhäuser/Springer Basel AG, Basel, 2011.

[13] L. Mari and L.F. Pessoa, The Ahlfors-Khas'minskii duality for nonlinear subequations, and geometric applications, preprint. 
[14] L. Mari and D. Valtorta, On the equivalence of stochastic completeness and Liouville and Khas'minskii conditions in linear and nonlinear settings, Trans. Amer. Math. Soc. 365 (2013), no. 9, 4699-4727.

[15] P. Mastrolia, M. Rigoli, and A. G. Setti, Yamabe-type equations on complete, noncompact manifolds, Progress in Mathematics, vol. 302, Birkhäuser/Springer Basel AG, Basel, 2012.

[16] H. Omori, Isometric immersions of Riemannian manifolds, J. Math. Soc. Japan 19 (1967), 205-214.

[17] S. Pigola, M. Rigoli, and A. G. Setti, A remark on the maximum principle and stochastic completeness, Proc. Amer. Math. Soc. 131 (2003), no. 4, 1283-1288 (electronic).

[18] _ Maximum principles on Riemannian manifolds and applications, Mem. Amer. Math. Soc. 174 (2005), no. 822, x+99.

[19] M. H. Protter and H. F. Weinberger, Maximum principles in differential equations, Springer-Verlag, New York, 1984, Corrected reprint of the 1967 original.

[20] P. Pucci, M. Rigoli, and J. Serrin, Qualitative properties for solutions of singular elliptic inequalities on complete manifolds, J. Differential Equations 234 (2007), no. 2, 507-543.

[21] P. Pucci and J. Serrin, Erratum to "the strong maximum principle revisited", J. Differential Equations 207 (2004), 226-227.

[22] _ The strong maximum principle revisited, J. Differential Equations 196 (2004), no. 1, 1-66.

[23] _ The maximum principle, Progress in Nonlinear Differential Equations and their Applications, 73, Birkhäuser Verlag, Basel, 2007.

[24] M. Rigoli and S. Zamperlin, "A priori" estimates, uniqueness and existence of positive solutions of Yamabe type equations on complete manifolds, J. Funct. Anal. 245 (2007), no. 1, 144-176.

[25] C. Villani, Optimal transport, old and new, Volume 338 of Grundlehren der mathematischen Wissenschaften, Springer, 2009.

[26] S.T. Yau, Some function-theoretic properties of complete Riemannian manifold and their applications to geometry, Indiana Univ. Math. J. 25 (1976), no. $7,659-670$. 
[27] J. W. York, Gravitational degrees of freedom and the initial-value problem, Phys. Rev. Lett. 26 (1971), 1656-1658.

[28] _ Covariant decompositions of symmetric tensors in the theory of gravitation, Ann. Inst. H. Poincaré Sect. A (N.S.) 21 (1974), 319-332.

E-mail addresses:

Marco Magliaro: marco.magliaro@gmail.com

Luciano Mari: mari@mat.ufc.br, luciano.mari@sns.it

Marco Rigoli: marco.rigoli55@gmail.com 